\title{
The Wiener and Zagreb Indices of Conjugacy Class Graph of the Dihedral Groups
}

\author{
${ }^{\mathbf{1}}$ Nur Idayu Alimon*, ${ }^{\mathbf{2}}$ Nor Haniza Sarmin and ${ }^{\mathbf{3}}$ Ahmad Erfanian \\ ${ }^{1,2}$ Department of Mathematical Sciences, Universiti Teknologi Malaysia \\ 81310 UTM Johor Bahru, Malaysia \\ ${ }^{3}$ Department of Pure Mathematics, Faculty of Mathematical Sciences, \\ Ferdowsi University of Mashhad, Mashhad, Iran \\ ${ }^{*}$ Corresponding author: nuridayualimon@yahoo.com
}

Article history

Received: 27 September 2018

Received in revised form: 21 January 2019

Accepted: 4 March 2019

Published on line: 1 April 2019

\begin{abstract}
Topological indices are numerical values that can be analysed to predict the chemical properties of the molecular structure which are computed for a graph related to groups. Meanwhile, the conjugacy class graph of $\mathrm{G}$ is defined as a graph with a vertex set represented by the non-central conjugacy classes of $G$. Two distinct vertices are connected if they have a common prime divisor. The main objective of this article is to find various topological indices including the Wiener index, the first Zagreb index and the second Zagreb index for the conjugacy class graph of dihedral groups of order $2 n$ where the dihedral group is the group of symmetries of regular polygon, which includes rotations and reflections. Many topological indices have been determined for simple and connected graphs in general but not graphs related to groups. In this article, the Wiener index and Zagreb index of conjugacy class graph of dihedral groups are generalized.
\end{abstract}

Keywords Wiener index; Zagreb index; conjugacy class graph; dihedral groups.

Mathematics Subject Classification $05 \mathrm{C} 12$.

\section{Introduction}

Graph theory has very wide applications in chemistry. For instance, a graph can be represented as a molecular structure where the vertices represent the atoms and the edges represent the bond between the atoms. In addition, various topological indices have been used to help chemists, mathematicians, clinicians, and many more in solving some problems related to chemistry or biology. It can also be used to determine the chemical or biological properties of some molecular structure. In [1], the Wiener index, the first Zagreb index and the second Zagreb index have been proven as tools for an efficient new drug design for anti-cancer and anti-HIV diseases.

The Wiener index was developed by the American Chemist, Harold Wiener in 1947 which defined as the sum of smallest distance between all vertices of the graph or the sum of half of the distances between every pair of vertices of graph, denoted by $W\left(\Gamma_{G}\right)$ [2]. Meanwhile, the

35:1 (2019) 51-57 | www.matematika.utm.my | eISSN 0127-9602 | 
first Zagreb index, $M_{1}\left(\Gamma_{G}\right)$ is the sum of squares of the degrees of the vertices, and the second Zagreb index, $M_{2}\left(\Gamma_{G}\right)$ is the sum of the products of the degrees of pair of adjacent vertices of the graph [3].

The presentation of the dihedral groups, $D_{2 n}$ is given in the following:

$$
D_{2 n}=\left\langle a, b \mid a^{n}=b^{2}=1, a^{b}=a^{-1}\right\rangle,
$$

where $n$ is a positive integer and $n \geq 3$.

\section{Preliminaries}

In this section, some basic concepts, definitions and previous results in group theory, graph theory and topological indices are included. The following are some definitions in group theory and graph theory that are used in this research.

Definition 1[4] Conjugacy Class of a

Let $a$ and $b$ be elements of a group $G$. The element of $a$ and $b$ are conjugate if $x a x^{-1}=b$ for some $x \in G$. The conjugacy classes of a is the set $c l(a)=\left\{x a x^{-1} \mid x \in G\right\}$ and the number of conjugacy classes of a is denoted by $k(G)$.

Definition 2 [5] Center of a Group

The center, $Z(G)$ of a group $G$ is the subset if elements in $G$ that commute with every element of $G$, written as, $Z(G)=\{a \in G \mid a x=x a, \forall x \in G\}$.

\section{Definition 3 [5] [6] Conjugacy Class Graph}

Let $G$ be a finite group. A conjugacy class graph, $\Gamma_{G}$ is a graph with the vertices $V=$ $\left(v_{1}, v_{2}, \ldots, v_{m}\right)$ represented by the non-central conjugacy classes of $G$. Two vertices $v_{1}$ and $v_{2}$ are connected if $\left|v_{1}\right|$ and $\left|v_{2}\right|$ have common prime divisor.

The following definitions stated the basic concepts that are used in computing the topological indices.

\section{Definition 4 [7] Degree of a Vertex}

The degree of a vertex $v$, deg $(v)$ is the number of edges, $|E(v)|$ at vertex $v$.

\section{Definition 5[7] Distance Between Two Vertices}

The distance, $d\left(v_{i}, v_{j}\right)$ of two vertices $v_{i}$ and $v_{j}$ is the shortest path from vertex $v_{i}$ to vertex $v_{j}$ where $i$ and $j$ denote the number of vertices.

\section{Definition 6 [8] The Wiener Index}

Let $v_{i}$ and $v_{j}$ be two distinct vertices where $i \neq j$ and $G$ be the connected graph with $m$ vertices. The Wiener index of a graph is defined as half of the sum of the distances between every pair of vertices of $G$, written as,

$$
W(G)=\frac{1}{2} \sum_{i=1}^{m} \sum_{j=1}^{m} d\left(v_{i}, v_{j}\right),
$$

where $d\left(v_{i}, v_{j}\right)$ is the shortest distance of $v_{i}$ and $v_{j}$. 
Definition 7 [9] The First Zagreb Index

Let $G$ be a connected graph. Then, the first Zagreb index is the sum of squares of the degrees of the vertices of $G$, written as,

$$
M_{1}(G)=\sum_{u \in V(G)} \operatorname{deg}(v)^{2},
$$

where $\operatorname{deg}(v)$ is the number of edges connected to vertex $v$.

\section{Definition 8 [9] The Second Zagreb Index}

Let $G$ be a connected graph. Then, the second Zagreb index is the sum of the product of the degrees of pairs of adjacent vertices of $G$, written as,

$$
M_{2}(G)=\sum_{u, v \in E(G)} \operatorname{deg}(u) \operatorname{deg}(v),
$$

where $u, v$ are the vertices on the edge connected them.

In addition, this research focuses on the dihedral groups of order $2 n$ where $n \geq 3$ and their conjugacy class graph. Based on the research done by Samaila et al. [10], the conjugacy classes for dihedral groups are divided into two cases which $n$ is odd and $n$ is even as stated in Proposition 1.

Proposition $1[10]$ Let $G$ be the dihedral groups, $D_{2 n}$ and $k(G)$ be the number of conjugacy classes of $G$. Then, the number of conjugacy classes of the dihedral groups are as follows :

$$
k(G)= \begin{cases}\frac{n+3}{2} & \text { if } n \text { odd } \\ \frac{n+6}{2} & \text { if } n \text { even },\end{cases}
$$

where $n=\frac{\left|D_{2 n}\right|}{2}$.

According to Mahmoud et al. in [11], the conjugacy class graph of all dihedral groups, $D_{2 n}$ can be divided into three parts, stated in the following proposition. This fact will be used in proving our main theorems.

Proposition 2 [11] Let $G$ be the dihedral group, $D_{2 n}=\left\langle a, b \mid a^{n}=b^{2}=1, a^{b}=a^{-1}\right\rangle$ of order $2 n$ where $n \geq 3, n \in \mathbb{N}$ and let $\Gamma_{G}$ be the conjugacy class graph of $G$. Then,

$$
\Gamma_{G}=\left\{\begin{array}{cl}
K_{\frac{n-1}{2}} \bigcup\{c l(b)\}, & \text { if } n \text { odd, } \\
K_{\frac{n+2}{2}}, & \text { if } n \text { and } \frac{n}{2} \text { even, } \\
K_{\frac{n-2}{2}} \bigcup K_{2}, & \text { if } n \text { even and } \frac{n}{2} \text { odd. }
\end{array}\right.
$$

\section{Results and Discussion}

Based on Proposition 2, only the second case involves a connected graph, while the other two cases give disconnected graphs. So, we only consider the case where $n$ and $\frac{n}{2}$ are even.

The Wiener index, the first Zagreb index and the second Zagreb index of the conjugacy class graphs of dihedral groups are given in the following three theorems. 
Theorem 1 Let $G$ be a dihedral group, $D_{2 n}=\left\langle a, b \mid a^{n}=b^{2}=1, a^{b}=a^{-1}\right\rangle$ of order $2 n$. Then, the Wiener index of the dihedral group is

$$
W\left(\Gamma_{G}\right)=(k(G)-|Z(G)|) k,
$$

where $n=4 k, k \in \mathbb{Z}^{+}, k(G)$ is the number of conjugacy classes in $G$, and $|Z(G)|$ is the number of central elements in $G$.

Proof First, we determine the number of vertices of the graph, namely $m$. From Definition 6 , the Wiener index is half of the sum of distances of every pair of vertices of the graph, written as,

$$
W\left(\Gamma_{G}\right)=\frac{1}{2} \sum_{i=1}^{m} \sum_{j=1}^{m} d\left(v_{i}, v_{j}\right) .
$$

By Definition 3 and Proposition 1, since the number of vertices of the conjugacy class graph of the dihedral groups is

$$
k(G)-|Z(G)|=\frac{n+6}{2}-2=\frac{n+2}{2},
$$

where $n=\frac{\left|D_{2} n\right|}{2}$ and there are two numbers of centers of the dihedral groups for $n$ is even, then the Wiener index of the conjugacy class graph of dihedral groups,

$$
\begin{aligned}
W\left(\Gamma_{G}\right) & =\frac{1}{2} \sum_{i=1}^{m} \sum_{j=1}^{m} d\left(v_{i}, v_{j}\right)=\frac{1}{2} \sum_{i=1}^{\frac{n+2}{2}} \sum_{j=1}^{\frac{n+2}{2}} d\left(v_{i}, v_{j}\right) \\
& =\frac{1}{2} \sum_{i=1}^{\frac{n+2}{2}}\left[d\left(v_{i}, v_{1}\right)+d\left(v_{i}, v_{2}\right)+\ldots+d\left(v_{i}, v_{\frac{n+2}{2}}\right)\right] \\
& =\frac{1}{2}\left[\left(d\left(v_{1}, v_{1}\right)+d\left(v_{2}, v_{1}\right)+\ldots+d\left(v_{\frac{n+2}{2}}, v_{1}\right)\right)+\ldots\right. \\
& \left.+\left(d\left(v_{1}, v_{\frac{n+2}{2}}\right)+d\left(v_{2}, v_{\frac{n+2}{2}}\right)+\ldots+d\left(v_{\frac{n+2}{2}}, v_{\frac{n+2}{2}}\right)\right)\right] \\
& =\frac{1}{2}\left[\left(\frac{n+2}{2}-1\right)\left(\frac{n+2}{2}\right)\right]=\frac{n(n+2)}{8} .
\end{aligned}
$$

Since we are only considering the case that $n$ and $\frac{n}{2}$ are even, this means $n=4 k, k \in \mathbb{Z}^{+}$. Hence, by substituting $n=4 k$ into the above equation and consider the number of vertices of the conjugacy class graph is the non-central conjugacy classes, then the Wiener index of the conjugacy class graph for the dihedral groups is

$$
W\left(\Gamma_{G}\right)=\frac{4 k(n+2)}{8}=\frac{k(n+2)}{2}=(k(G)-|Z(G)|) k .
$$

Theorem 2 Let $G$ be a dihedral group, $D_{2 n}=\left\langle a, b \mid a^{n}=b^{2}=1, a^{b}=a^{-1}\right\rangle$ of order $2 n$. Then, the first Zagreb index of dihedral group is

$$
M_{1}\left(\Gamma_{G}\right)=n\left(W\left(\Gamma_{G}\right)\right),
$$

where $n=4 k, k \in \mathbb{Z}^{+}$and $W\left(\Gamma_{G}\right)$ is the Wiener index of the conjugacy class graph of $G$. 
Proof From Definition 7, the first Zagreb index is the squares of the degrees of each vertices of the graph, written as,

$$
M_{1}\left(\Gamma_{G}\right)=\sum_{v_{i} \in V(G)} \operatorname{deg}\left(v_{i}\right)^{2} .
$$

By Definition 3 and Proposition 1, since the conjugacy class graph, $\Gamma_{G}$ only considered the noncentral conjugacy classes to be its vertices, then $1 \leq i \leq \frac{n+2}{2}$ which $k(G)-|Z(G)|=\frac{n+6}{2-1}=\frac{n+2}{2}$. Now, the first Zagreb index of the conjugacy class graph is as follows :

$$
\begin{aligned}
M_{1}\left(\Gamma_{G}\right) & =\sum_{i=1}^{\frac{n+2}{2}} \operatorname{deg}\left(v_{i}\right)^{2}=\operatorname{deg}\left(v_{1}\right)^{2}+\operatorname{deg}\left(v_{2}\right)^{2}+\ldots+\operatorname{deg}\left(v_{\frac{n+2}{2}}\right)^{2} \\
& =\left[\left(\frac{n+2}{2}-1\right)^{2}+\left(\frac{n+2}{2}-1\right)^{2}+\ldots+\left(\frac{n+2}{2}-1\right)^{2}\right] \\
& =\frac{n+2}{2}\left(\frac{n+2}{2}-1\right)^{2}=(k(G)-|Z(G)|)\left(\frac{n}{2}\right)^{2} .
\end{aligned}
$$

The first Zagreb index only applicable for the second case of the conjugacy class graph as stated in Proposition 2. By substituting $n=4 k$ where $k \in \mathbb{Z}^{+}$into the above formula, then the first Zagreb index of the conjugacy class graph of the dihedral groups is

$$
M_{1}\left(\Gamma_{G}\right)=4 k^{2}(k(G)-|Z(G)|)=4 k W\left(\Gamma_{G}\right)=n W\left(\Gamma_{G}\right),
$$

where $n=\frac{\left|D_{2} n\right|}{2}, k(G)$ is the number of conjugacy classes, $|Z(G)|$ is the order of the center of the dihedral groups and $W\left(\Gamma_{G}\right)$ is the Wiener index of the conjugacy class graph of some dihedral groups.

Theorem 3 Let $G$ be a dihedral group, $D_{2 n}$ of order $2 n$. Then, the second Zagreb index of dihedral group is

$$
M_{2}\left(\Gamma_{G}\right)=k\left(M_{1}\left(\Gamma_{G}\right)\right),
$$

where $n=4 k, k \in \mathbb{Z}^{+}$and $M_{1}\left(\Gamma_{G}\right)$ is the first Zagreb index of the conjugacy class graph of $G$.

Proof By Definition 8, the second Zagreb index is the sum of the product of the degrees of pairs of adjacent vertices said $u$ and $v$ of $G$, written as,

$$
M_{2}\left(\Gamma_{G}\right)=\sum_{u, v \in E(G)} \operatorname{deg}(u) \operatorname{deg}(v) .
$$

Since we only considered the second case of Proposition 2, then the graph involved is a complete graph. The number of edges for the complete graph, $K_{m}$ is $\frac{m(m-1)}{2}$, where $m$ is the number of vertices.

Since the vertices of the conjugacy class graph is the non-central conjugacy classes where $k(G)-|Z(G)|=\frac{n+2}{2}, n=\frac{\left|D_{2} n\right|}{2}$, then we can rewrite the above formula by substituting $\frac{n+2}{2}$ into $m$. So that, the number of edges of the conjugacy class graph (second case of Proposition 2 ) of dihedral groups is $\frac{n(n+2)}{8}$ and the degree of each vertex is $\frac{n+2}{2-1}=\frac{n}{2}$ since it is the complete 
graph. Hence, the second Zagreb index of the conjugacy class graph for the dihedral group is written as follows :

$$
\begin{aligned}
M_{2}\left(\Gamma_{G}\right) & =\sum_{i=1}^{\frac{n(n+2)}{8}} e_{i}=e_{1}+e_{2}+e_{3}+\ldots+e_{\frac{n(n+2)}{8}} \\
& =\left(\frac{n}{2}\right)\left(\frac{n}{2}\right)\left(\frac{n(n+2)}{8}\right)=\frac{n^{3}}{16}\left(\frac{n+2}{2}\right) \\
& =\frac{n^{2}}{4} k(k(G)-|Z(G)|) \\
& =k n W\left(\Gamma_{G}\right)=k M_{1}\left(\Gamma_{G}\right)
\end{aligned}
$$

where $e_{i}$ is the $i^{t h}$ edge of the conjugacy class graph, $n=4 k, k \in \mathbb{Z}^{+}, n=\frac{\left|D_{2} n\right|}{2}$, and $M_{1}\left(\Gamma_{G}\right)$ is the first Zagreb index of the conjugacy class graph of the dihedral groups.

\section{Conclusion}

The computation of topological indices only valid for the connected graph. Hence, only the second case of conjugacy class graph of the dihedral groups in Proposition 2 is considered in this paper. Since the conjugacy class graph of dihedral groups is a complete graph, then the generalization of the Wiener index and the Zagreb index of a complete graph are used to obtain those indices. In conclusion, the first Zagreb index is the multiple of $2 n$ of the Wiener index for the conjugacy class graph of some dihedral groups where $n=4 k, k \in \mathbb{Z}^{+}$. Meanwhile, the second Zagreb index of the conjugacy class graph of some dihedral groups is the multiple of $k$ of its first Zagreb index.

The results obtained can help the chemists or other related industry to apply the findings in order to analyze the chemical or biological properties of specific molecular structure. In future, the generalization of different types of graphs and groups will be determined.

\section{Acknowledgments}

The authors would like to express their appreciation for the support of the sponsors; Ministry of Higher Education (MOHE) Malaysia and Research Management Center (RMC), Universiti Teknologi Malaysia (UTM) Johor Bahru for the financial funding through the Research University Grant (GUP) Vote No. 13HJ9 and 13J82. The first author is also indebted to Universiti Teknologi Malaysia (UTM) for her Zamalah Scholarship.

\section{References}

[1] Senbagamalar, J. On Topological Indices of Molecular Graphs. PhD Thesis. 2014.

[2] Vijayabarathi, A. and Arijaneyulu, G.S.G. N. Wiener index of a graph and chemical applications. International Journal of Chem Tech Research. 2013. 1847-1853.

[3] Das, K.C., Xu, K. and Nam, J. Zagreb indices of graphs. Frontier Mathematics China. 2015. 10:567-582.

[4] Hall, J. The Theory of Groups. American Mathematical Society. 1967. 
[5] Woods, C. My favourite application using graph eigenvalues : Graph energy. Available online. 2013. 17:535-538.

[6] Bertram, E.A., Herzog, M. and Mann, A. On a graph related to conjugacy classes of groups. Bulletin of the London Mathematical Society. 1990. 22:569-575.

[7] Bondy, J., and Murty, U. Graph Theory with Applications. The Macmillan Press LTD. 1976.

[8] Graovac, A., and Pisanski, T. On the Wiener index of a graph. Journal of Mathematical Chemistry. 1991. 8:53-62.

[9] Gutman, I., and Trinajstić, N. Graph theory and molecular orbitals : Total $\pi$-electron energy of alternant hydrocarbons. Chemical Physics Letter. 1972. 17:535-538.

[10] Samaila, D., Abba, B.I. and Pur, M. P. On the conjugacy classes, centers, representation of the groups $S_{n}$ and $D_{n}$. International Journal of Pure and Applied Sciences and Technology. 2013. 15:87-95.

[11] Mahmoud, R., Sarmin, N.H. and Erfanian, A. The conjugacy class graph of some finite groups and its energy. Malaysian Journal of Fundamental and Applied Sciences. 2017. 13:659-665. 